\title{
The CJ12 parton distributions
}

\section{A. Accardi*}

Hampton U. and Jefferson Lab

E-mail: accardiejlab.org

\section{J. F. Owens}

Florida Sate University

\section{W. Melnitchouk}

Jefferson Lab

Three new sets of next-to-leading order parton distribution functions (PDFs) are presented. These are determined by global fits to a wide variety of data for hard scattering processes. The analysis includes target mass and higher twist corrections needed for the description of deep inelastic scattering data at large $x$ and low $Q^{2}$, and nuclear corrections for deuterium targets. The PDF sets correspond to three different models for the nuclear effects, and provide a more realistic uncertainty range for the $d$ quark PDF compared with previous fits. Applications, in particular to weak boson production at colliders, are also be discussed.

XXI International Workshop on Deep-Inelastic Scattering and Related Subject - DIS2013,

22-26 April 2013

Marseilles, France

*Speaker. 
Introduction. Plentiful DIS data exist from fixed-target experiments at large $x$ and low scale $Q^{2}$, which is typically excluded in global QCD fits by imposing cuts on the invariant mass $W \gtrsim 3.8$ $\mathrm{GeV}$ in order to exclude the region where higher-twists and other subleading in $1 / Q^{2}$ effects are important. However, there are many reason why one should try and obtain precise PDFs at large $x$. For example, they are necessary to reduce the uncertainty on the QCD background in searches for new physics in high $p_{T}$ spectra or large mass at the LHC. On the other end of the energy spectrum, the behavior of ratios like $d / u$ or $\Delta u / u$ and $\Delta d / d$ at $x \rightarrow 1$ can discriminate between models of the non perturbative structure of the nucleon. Finally, the uncertainty in the extraction of the helicity gluon PDF at the smallest $x$, which can be reached in forward particle production in $p+p$ collisions, is fundamentally limited by the PDF uncertainty on the large- $x$ quark against which the gluon scatters.

While these are certainly noble and necessary goals, the most interesting aspect of large- $x$ global fits is their ability to connect particle physics at high-energy with hadronic and nuclear physics at medium energy [1]. For example, data on $W$ and $Z$ forward rapidity boson production at Tevatron and the LHC, which can reach large values of $x$, can constrain the extrapolation to $x=1$ of the down-quark to up-quark ratio in the proton, and (with enough statistical precision) indicate which nonperturbative proton structure model best captures the effects of confinement on hadron structure. Likewise, but less obviously, global PDF fits can be used as a tool to study the structure of the nucleus and the differences between bound and free protons, for which current models display large theoretical uncertainties. This is possible, e.g., by exploiting the interplay of Deep Inelastic Scattering (DIS) data in deuteron targets, which allow one to extract a nuclear model dependent $d$ quark distribution at large $x$ [2-4], and weak interaction processes on proton targets, which also depend on the $d$ quark but are naturally free from nuclear effects. Global fits can thus turn detectors such as CDF at the Tevatron or LHCb at the LHC into nuclear physics experiments complementing more traditional lower energy ones on fixed nuclear targets, such as at Jefferson Lab.

The CTEQ-Jefferson Lab global PDF fits. The CTEQ-Jefferson Lab (CJ) collaboration was formed with the aim to include subleading $O\left(1 / Q^{2}\right)$ effects and large- $x$ corrections in the perturbative calculations utilized in global PDF fits - initially, target mass corrections and parametrized higher-twist terms - and to investigate the effect of relaxing the invariant mass cut to just inside the resonance region, $W>1.7 \mathrm{GeV}$. The resulting fits [2-4] have culminated in the release of the "CJ12" PDF sets, valid in the whole $10^{-5} \lesssim x \lesssim 0.9$ range. These are available on the CTEQ-JLab web page [5] and as part of the LHAPDF interface [6]. The fits were performed at NLO in the zero mass variable flavor number scheme, with $\alpha_{s}$ fixed to the the particle data book's world average. Full heavy quark treatments, fits of the strong coupling constant and inclusion of the available LHC data will be considered next.

The CJ PDFs have been shown to be stable with the weaker cuts, and the increase of about 1000 DIS data points has led to significantly reduced errors, in particular up to $40-60 \%$ on the $d$ quark at large $x \gtrsim 0.6$, where precise data sensitive to this distribution are otherwise scarce [2]. Since $d$ quark flavor separation at large $x$ is currently almost entirely dependent on DIS on deuteron target, corrections for nuclear Fermi motion and binding effects are included by convoluting the nucleon structure functions with a smearing function calculated from the deuteron wave function. As the $u$ quark is well constrained by data on proton targets, the $d$ quark becomes directly sensitive to the 

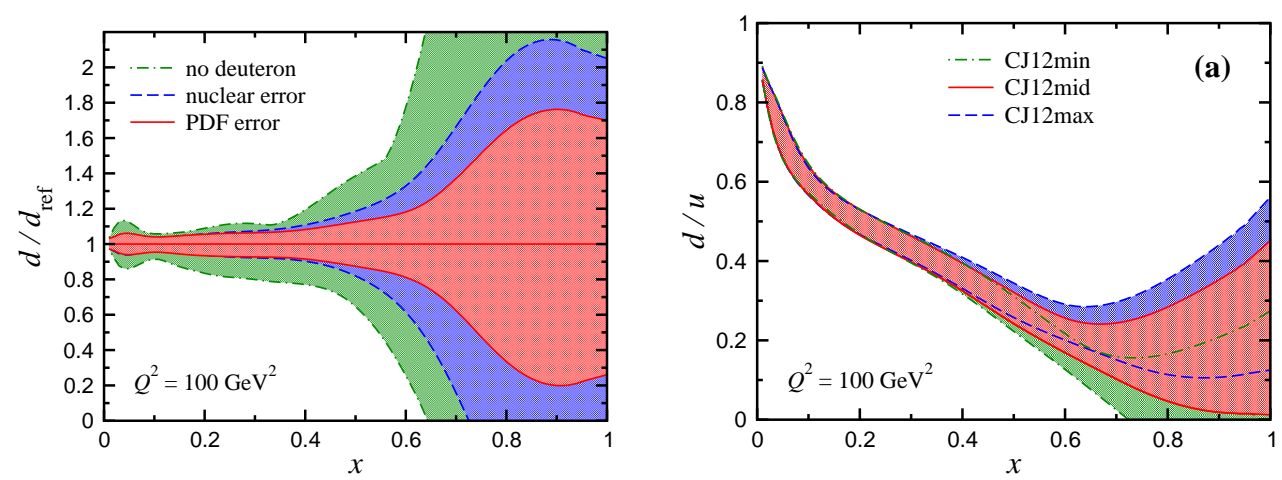

Figure 1: Left: PDF uncertainties for the CJ12mid $d$ quark compared with the total uncertainty including nuclear corrections and with a fit excluding all Deuteron data relative to the CJ12mid set. Right: $d / u$ ratio for the CJ12min, CJ12mid and CJ12max PDFs. Plots taken from Ref. [4].

nuclear corrections. The effect [2] is a large suppression at high $x$, and a mild but non-negligible increase at intermediate $x$, still inside the "safe" region defined by the larger $W$ cut discussed above, as recently confirmed by Ball et al. [7].

References $[3,4]$ have quantified the uncertainties on the $d$ quark arising from theoretical modeling of nuclear corrections, or "nuclear uncertainties" in short. These range from mild, corresponding to the hardest of the modern deuteron wave functions (WJC-1) coupled to a $0.3 \%$ nucleon off-shellness, to strong, corresponding to the softest wave function (CD-Bonn) and a large, $2.1 \%$ nucleon off-shellness, with a central value corresponding to the AV18 wave function and a $1.2 \%$ off-shellness. The resulting PDFs are labeled CJ12min, CJ12max, and CJ12mid, respectively. The uncertainties in the CJ12 $d$ quark distribution are illustrated in Fig. 1 left. The red band is the PDF error calculated with the Hessian method and a tolerance factor $T=10$. The blue band represents the theoretical nuclear uncertainty, obtained as an envelope of the CJ12min and CJ12max fits, and is of the same order of magnitude as the PDF error. The green band is the PDF error in a fit without deuterium data and exceeds the nuclear uncertainties. This demonstrates the usefulness of the deuterium data, even in the presence of the nuclear uncertainties that its use introduces.

A further source of theoretical uncertainty was investigated in Ref. [3, 4], where a more flexible valence $d_{v}$ quark parametrization at large- $x$ with a small admixture of the valence $u_{v}$ PDF was used:

$$
d_{v}(x) \rightarrow d_{v}^{\prime}(x)=a_{0}^{d}\left[d_{v}(x) / a_{0}^{d}+b x^{c} u_{v}(x)\right],
$$

where $a_{0}^{d}$ is the $d$ quark normalization, and $b$ and $c$ are two additional parameters. The result is that the $d / u$ ratio at large $x \rightarrow 1$ can now span the range $(0, \infty]$ rather than being limited to either 0 or $\infty$ as in all other PDF fits. A finite, nonzero value of this ratio is in fact expected in several nonperturbative models of hadron structure, see Ref. [8]. It is also required from a purely practical point of view because it avoids potentially large biases on the fitted $d$ quark PDF, as discussed in more detail later.

The ratios of the $d$ to $u$ PDFs for the three CJ12 sets are shown in Fig. 1 right. These are constrained up to $x \approx 0.8$ by the enlarged data set considered in the CJ fits, but can be confidently extrapolated to $x=1$ thanks to the modified $d$ quark parametrization (1). It was found that

$$
d / u \underset{x \rightarrow 1}{\longrightarrow} 0.22 \pm 0.20(\mathrm{PDF}) \pm 0.10(\mathrm{nucl})
$$


where the first error is from the PDF fits and the second is from the nuclear correction models. These values encompass the 0-0.5 range of available theoretical predictions [8]. However, a relatively modest improvement in statistical precision and reduction of nuclear uncertainty would allow one to restrict the range of allowable physical mechanisms.

The same nuclear uncertainty also affects forward rapidity observables or production of large mass particles [9]. For example, the nuclear uncertainty becomes relevant for $W$ production at rapidity larger than 2 at the Tevatron, and larger than 3.5 at the LHC. For particles of heavier mass such as as $W^{\prime}$ and $Z^{\prime}$ bosons the nuclear uncertainty in the production cross section at large $x$ may become larger than $20 \%$ above the lower mass limit of about $2.5 \mathrm{TeV}$ set by recent LHC data [10]. One can then appreciate how nuclear uncertainties and other large- $x$ theoretical uncertainties may significantly affect the interpretation of signals of new particles and an accurate measurement of their properties, which require a precise determination of QCD backgrounds.

Constraining nuclear uncertainties with proton targets. As discussed in the introduction, global QCD fits allow for the intriguing possibility to combine deuteron and proton target data to obtain experimental constraints on nuclear models, and at the same time fully utilize the available statistics of the nuclear target data. For example, the directly reconstructed $W$ asymmetry at Tevatron is very sensitive to nuclear corrections at $y \gtrsim 2$ [9]. A comparison with the very precise CDF data as well as the total $\chi^{2}$ values in the CJ12 fits suggest that nuclear effects are somewhere between the minimum and central ones considered in the CJ12min and CJ12mid fits, respectively. This was also confirmed in a similar analysis by Martin et al. [11], where the nuclear corrections were fitted to data rather than calculated in a microscopic model. On the phenomenological side, this result disfavors nonperturbative proton models based on the SU(6) spin-flavor symmetry, which predict $d / u \rightarrow 1 / 2$ as $x \rightarrow 1$. More importantly, it exemplifies the power of global fits in combining data from across the board, and is the first step in establishing the experimental foundation that was until now missing for a qualitative jump in our ability to theoretically understand and describe high-energy processes in nuclei - and this without even utilizing nuclear targets!

The $W$ charge asymmetry at CDF unfortunately seems the only observable that currently has this potential. The lepton asymmetry has insufficient large $x$ coverage due to decay vertex smearing, while $Z$ rapidity distributions from $\mathrm{CDF}$ and $\mathrm{D} \varnothing$ have insufficient precision [4]. At the LHC one would need measurements with better than $10 \%$ precision at $y \gtrsim 3.5$, namely, at the edge of the $\mathrm{LHCb}$ acceptance. The potential of large rapidity reconstructed $W$ and $Z$ measurements in $p+p$ collisions at RHIC should also be explored. Finally, parity-violating DIS scattering provides another way to separate $u$ and $d$ quarks. Recent measurements of $F_{2,3}^{\gamma Z}$ at HERA and the planned large- $x$ measurements at the upgraded Jefferson Lab accelerator will provide additional leverage to constrain nuclear corrections.

Theoretical biases at $x \rightarrow 1$. The importance of using a more flexible $d$-quark parametrization than in traditional fits cannot be overemphasized. Figures 2 and 3 show a comparison of the CJ12mid fits to a fit obtained with the standard $W>3.5 \mathrm{GeV}$ cut, and a fit obtained with $W>1.7$ $\mathrm{GeV}$ but standard $d$-quark parametrization, namely, $b=c=0$ in Eq. (1).

In Figure 2, the ratios of the up, down quarks and the gluons in the 2 new fits to the CJ12mid fit are presented with a tolerance $T=1$ for clarity of presentation. The fit using standard parametriza- 


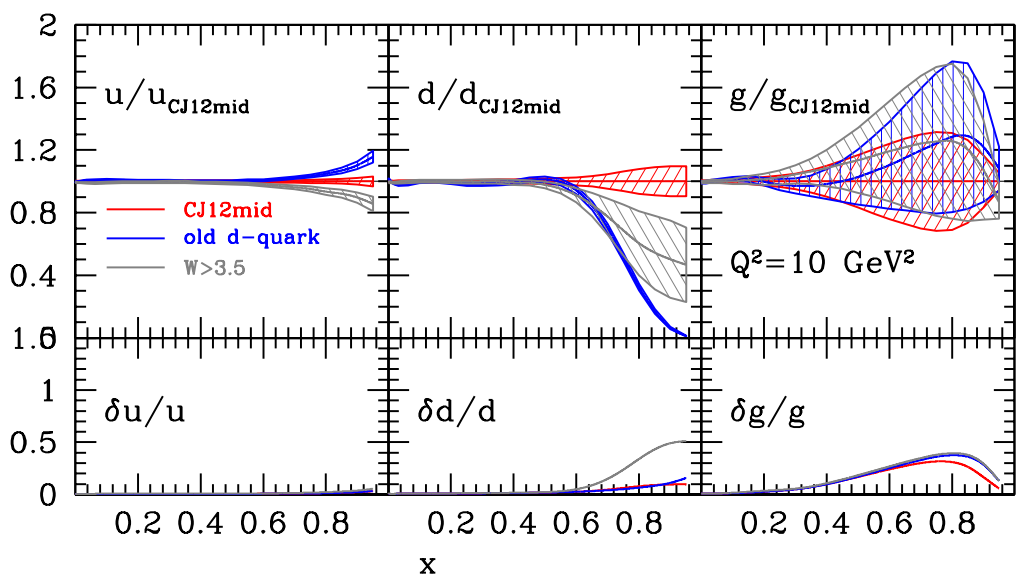

Figure 2: Comparison of PDF obtained with a conventional $W>3.5 \mathrm{GeV}$ cut, and using the old $d_{v}$ quark parametrization with $W>1.7 \mathrm{GeV}$ to the $\mathrm{CJ} 12$ mid set. A tolerance $T=1$ is used for clarity.

tion starts deviating from the CJ12mid fit at $x \gtrsim 0.5$. This behavior is clearly not data driven (it starts already inside the covered kinematic range) but forced by the assumed functional form for $d \propto(1-x)^{a_{d}}$ rather than the more flexible parametrization (1). The deep dive of the standard $d$ quark towards 0 can be compensated in the fit by an increased $u$ quark distribution, due to the correlation induced by large- $x$ DIS data.

A deeper insight can be obtained by looking at the at the $d / u$ plots in Fig 3. In this case the standard parametrization forces $d / u$ to take either a value of 0 or $\infty$. In contrast the extended $d^{\prime}$ parametrization allows the whole range $(0, \infty]$. In the CJ12 fit, the data does not seem to warrant the behavior of the standard parametrization, which lies at the edge of the PDF error band of the CJ12mid fit. In summary, Not only does the standard $d$ parametrization underestimates the central fitted value at $x \gtrsim 0,5$, but also and crucially it underestimates the PDF uncertainty as well as the nuclear uncertainty.

Other potential biases exist at large $x$. These are related to theoretical corrections not yet included included in the perturbative QCD calculations utilized in the CJ12 analysis, e.g., large- $x$ resummation, jet mass corrections and higher-order terms in the perturbative expansion. However,
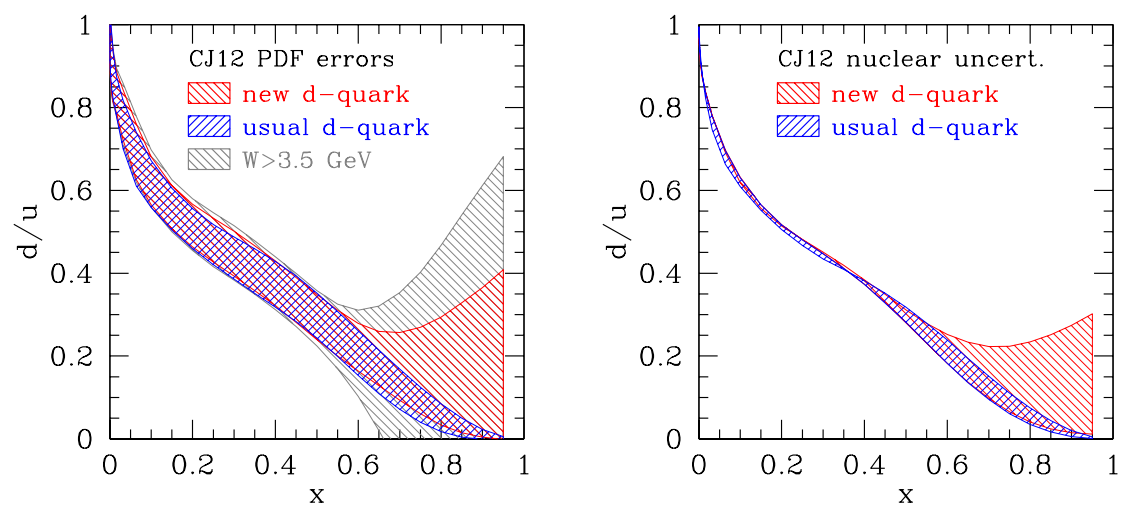

Figure 3: PDF errors with tolerance $T=10$ and nuclear uncertainties for the fits of the previous Figure. 
these either scale as an inverse power of $Q^{2}$ or resemble one at low $Q^{2}$ and will mainly affect the extraction of the HT term, leaving the leading-twist PDFs largely unchanged as it happens for the model dependence of target mass corrections [2]. This makes the choice of parametrization possibly the largest but typically neglected theoretical bias in the determination of the $d$-quark distribution at large $x$. A full theoretical unbiasing should be pursued by generalizing the $d^{\prime}$ quark functional form adopted in the $\mathrm{CJ} 12$ fits and investigating how solid the related quantitative extraction of $d$-quark errors at $x \rightarrow 1$ is.

Conclusions. The recent studies by the CTEQ-Jefferson Lab collaboration, culminating in the public CJ12 PDF release [4], have demonstrated the intimate interconnection of hadronic, nuclear and high-energy physics. Namely, global QCD fits have become capable of constraining theoretical models of nuclear corrections in the deuteron. Not only will this reduce the nuclear uncertainty on the fitted PDFs with important phenomenological consequences on physics ranging from nonperturbative proton structure to beyond the standard model interactions, it will also provide a new avenue for progress in the theoretical understanding of high-energy processes involving nuclei, using weak interactions on proton targets from Jefferson Lab to the LHC.

Acknowledgments: This work was supported by the DOE contract No. DE-AC05-06OR23177, under which Jefferson Science Associates, LLC operates Jefferson Lab, and by the DOE contracts DE-SC008791 and DE-FG02-97ER41922.

\section{References}

[1] A. Accardi, "PDF uncertainties at large $x$ and gauge boson production," PoS ConfinementX (2012) 227.

[2] A. Accardi, M. E. Christy, C. E. Keppel, P. Monaghan, W. Melnitchouk, J. G. Morfin and J. F. Owens, New parton distributions from large-x and low- $Q^{2}$ data, Phys. Rev. D 81 (2010) 034016.

[3] A. Accardi, W. Melnitchouk, J. F. Owens, M. E. Christy, C. E. Keppel, L. Zhu and J. G. Morfin, Uncertainties in determining parton distributions at large $x$, Phys. Rev. D 84 (2011) 014008.

[4] J. F. Owens, A. Accardi and W. Melnitchouk, Global parton distributions with nuclear and finite- $Q^{2}$ corrections, Phys. Rev. D 87 (2013) 094012.

[5] The CTEQ-Jefferson Lab (CJ) collaboration website, http: / / www . jlab.org/cj.

[6] The CJ12 PDFs are also available in the LHAPDF interface version 5.8.9 and higher, https://lhapdf.hepforge.org/.

[7] R. D. Ball et al. [ The NNPDF Collaboration], Phys. Lett. B 723 (2013) 330 [arXiv:1303.1189 [hep-ph]].

[8] R. J. Holt and C. D. Roberts, Distribution Functions of the Nucleon and Pion in the Valence Region, Rev. Mod. Phys. 82 (2010) 2991

[9] L. T. Brady, A. Accardi, W. Melnitchouk and J. F. Owens, Impact of PDF uncertainties at large x on heavy boson production, JHEP 06 (2012) 019.

[10] G. Dissertori, PoS (Confinement X) 011 (2012).

[11] A. D. Martin et al., Extended Parameterisations for MSTW PDFs and their effect on Lepton Charge Asymmetry from W Decays, Eur. Phys. J. C 73 (2013) 2318. 